\title{
EDITORIAL \\ Toward the elimination of bias in Pediatric Research
}

\author{
Pediatric Research (2019) 86:680-681; \\ https://doi.org/10.1038/s41390-019-0583-5
}

There is increasing evidence that unconscious bias can affect realworld decision-making processes in publication just as in many other fields. ${ }^{1}$ In response, the editorial board of Pediatric Research is working to investigate and reduce the bias in the publication acceptance rates in order to preserve the integrity of the peer review process and publication. As news items have suggested that gender bias is a major problem in academia, ${ }^{2}$ we reviewed papers submitted between 1 November 2017 and 9 August 2018 to Pediatric Research. Encouragingly, we found that the acceptance rates of manuscripts were not significantly different between corresponding authors who were male or female. However, we incidentally uncovered a higher rejection rate in the manuscripts where the corresponding author had a name that could not be identified as either male or female and did not have a picture on their website so that we could identify their gender. ${ }^{3}$ It is important to point out that we do not know the reason for this, but its identification is the first step to further exploration, including assessing whether unconscious bias may play a role.

Diversity, including gender diversity leads to better science and may contribute to the "innovation dividend." ${ }^{4}$ Many institutions are tackling unconscious bias through training. UK and Irish universities have to fulfill specific requirements under the Athena SWAN (Scientific Women's Academic Network) Charter, a formalized agreement that acknowledges and rewards good practices in higher education and research institutions to improve ethnic, racial, and gender equality: representation, progression, and success for all. ${ }^{2,5}$ Universities and medical institutions worldwide have encouraged unconscious bias training including both the areas of equality and diversity. Some courses are available online (https://www.tcd.ie/equality/training/lead-online-training/; http://kirwaninstitute.osu.edu/implicit-bias-training/; https://www. youtube.com/watch?v=v01SxXui9XQ\&t=62s\&app=desktop; https://www.aamc.org/members/leadership/catalog/178420/ unconscious_bias.html) making compliance relatively easy.

In a subsequent correspondence to our editorial, Jagsi et al. ${ }^{6}$ pointed out that the medical academy still has a long way to go in eliminating bias in publication rates. Pediatric Research agrees with this conclusion and would like to describe the efforts being taken to eliminate bias. We feel it is important to assess a more complete picture of potential gender bias by having essential data. Our actions are as follows:

First, the managing editor is collecting gender information of the first, last, and corresponding authors, as well as authors applying for and receiving our early career investigator publication award. Using these data, in the next few years, we will re-examine gender bias in acceptance rates at Pediatric Research for first and senior authors and early career investigators separately. This information will only be seen by the managing editor and is not revealed to the editors or reviewers as it is for internal use only.

Second, we are encouraging current members of the editorial board to participate in an unconscious bias course. All new members of the editorial board will be required to take an online unconscious bias course as will our editorial apprentices.

Third, we are analyzing the reasons for rejection of manuscripts. Rejection can occur as Reject without Review (rejected before peer review), Reject (reviewers found a fatal flaw in the paper), or Reject and Resubmit (Reject and resubmit is used when the reviewers feel that revisions will take longer than the normal time for a resubmission but are enthusiastic about the manuscript). While we are gathering data to determine if papers that are novel and have merit are being rejected with repairable flaws, this analysis will also allow us to determine whether, unconsciously, gender plays a role in any of the "rejection" categories we have.

Many articles that are rejected come from low or very low resource countries. In some cases, these may be more appropriately handled by a "country advisor," someone from the country of origin that could assist in developing the manuscript to a publishable form. ${ }^{7}$ We currently refer papers with merit but need attention to a Springer-Nature English editing resource to deal with language issues for manuscripts we wish to publish. Pediatric Research will identify first or last authors from accepted manuscripts from these nations in our new feature on Global Pediatric Research Community. They will be featured each month in a commentary and invited to join a resource to other authors in their countries who wish to publish in Pediatric Research.

However, many manuscripts will not be acceptable to Pediatric Research. There are many reasons for rejection. Some do not meet our rigorous scientific or ethical standards and many are simply beyond the scope of the topics we cover. In an effort to assist these latter manuscripts still to be published, we are developing a transfer desk with Springer-Nature, a formal mechanism to reroute these manuscripts to journals more suitable for their scope, either content-wise or articles that do not have a global impact.

In sum, Pediatric Research is combating bias, including unconscious bias, on multiple levels. Stay tuned for updates!

Cynthia Bearer ${ }^{1}$, Carlo Agostoni ${ }^{2}$, Kanwaljeet J. S. Anand ${ }^{3}$, Namasivayam Ambalavanan ${ }^{4}$, Vineet Bhandari ${ }^{5}$, Joseph M. Bliss, Frank Bloomfield ${ }^{7}$, Sonia L. Bonifacio ${ }^{8}$, Irina Buhimschi ${ }^{9}$, Maria Roberta Cilio ${ }^{10}$, Max Coppes ${ }^{11,12}$, Steven J. Czinn ${ }^{13}$ ', Afif EL-Khuffash ${ }^{14}$, Nicholas Embleton ${ }^{15}$

Ursula Felderhoff-Müser ${ }^{16}$, Donna M. Ferriero ${ }^{17}$, Todd Florin ${ }^{18}$, Elena Fuentes-Afflick ${ }^{19}$, William Gardner ${ }^{20}$, Sidney M. Gospe Jr. ${ }^{21}{ }^{\prime}$ Alistair Gunn ${ }^{22}$, Pierre Gressens ${ }^{23}$, Dino Guissani ${ }^{24}$, Nadja Haiden ${ }^{25}$, Marissa Hauptman ${ }^{26}$, Kwang Sik Kim ${ }^{27}$, Mark Klebanoff ${ }^{28}$ ', Peter Lachman ${ }^{29}$, Bruce Lanphear ${ }^{30}$, Seza Ozen ${ }^{31}$, Charles Roehr ${ }^{32}$ ', Damian Roland ${ }^{33}$, Norman Rosenblum ${ }^{34}$, Margaret Schwarz ${ }^{35}$, Annamaria Staiano ${ }^{36}$, Annemarie Stroustrup ${ }^{37^{\prime}}$ Enza Maria Valente ${ }^{38}$, Dee Wilson-Costello ${ }^{39}$, James Wynn ${ }^{40}$ and Eleanor Molloy ${ }^{41}$

${ }^{1}$ Department of Pediatrics, University of Maryland, Baltimore, MD, USA; ${ }^{2}$ Department of Pediatrics, IRCCS Ospedale Maggiore, Policlinico, University of Milan, Milan, Italy; ${ }^{3}$ Stanford University School of Medicine, Stanford, CA, USA; ${ }^{4}$ University of Alabama at Birmingham, Birmingham, AL, USA; ${ }^{5}$ Department of Neonatology, St.

Christopher's Hospital for Children, Philadelphia, PA, USA; ${ }^{6}$ Brown University, Women and Infants Hospital, Providence, RI, USA; ${ }^{7}$ Liggins 
Institute, University of Auckland, Auckland, New Zealand; ${ }^{8}$ Neonatal and Developmental Medicine, NICU, Stanford University School of Medicine, Palo Alto, CA, USA; ${ }^{9}$ Center for Perinatal Research, Nationwide Childrens Hospital, Columbus, OH, USA; ${ }^{10}$ Department of Neurology, University of California, San Francisco, San Francisco, CA, USA; ${ }^{11}$ Pediatrics; UNSOM - Pediatrics, University of Nevada School of Medicine, Reno, NV, USA; ${ }^{12}$ Renown Health, Renown Children's Hospital, Reno, NV, USA; ${ }^{13}$ University of Maryland School of Medicine, Baltimore, MD, USA; ${ }^{14}$ The Rotunda Hospital, Neonatology, The Royal College of Surgeons in Ireland, Dublin, Ireland; ${ }^{15}$ Newcastle Hospitals NHS Foundation Trust, Royal Victoria Infirmary, Newcastle upon Tyne, UK; ${ }^{16}$ Department of Pediatrics I, University Hospital Essen, University of Duisburg-Essen, Essen, Germany; ${ }^{17}$ Weill Institute for Neurosciences, San Francisco, CA, USA; ${ }^{18}$ Cincinnati Children's Hospital Medical Center, Pediatrics - Pediatric Emergency Medicine, Cincinnati, $\mathrm{OH}, \mathrm{USA} ;{ }^{19}$ Department of Pediatrics, San Francisco General Hospital, University of California, San Francisco, CA, USA; ${ }^{20}$ CHEO Research Institute, Ottawa, ON, Canada; ${ }^{21}$ Seattle Children's Hospital, Neurology, Seattle, WA, USA; ${ }^{22}$ Dept of Physiology, University of Auckland, Auckland, New Zealand; ${ }^{23}$ Hopital Robert Debre, Paris, France; ${ }^{24}$ Physiology Development and Neuroscience, University of Cambridge, Cambridge, UK; ${ }^{25}$ Department of Clinical Pharmacology, Medical University of Vienna, Vienna, Austria;

${ }^{26}$ General Pediatrics, Children's Hospital Boston, Boston, MA, USA;

${ }^{27}$ Pediatrics Infectious Diseases, Baltimore, MD, USA; ${ }^{28}$ Nationwide Children's Hospital, Center for Perinatal Research, Columbus, $\mathrm{OH}$, USA; ${ }^{29}$ International Society for Quality in Health Care, Dublin, Ireland; ${ }^{30}$ Simon Fraser University, Burnaby, BC, Canada; ${ }^{31}$ Hacettepe Univeristy School of Medicine, Pediatrics, Ankara, Turkey; ${ }^{32} \mathrm{John}$ Radcliffe Hospital, Newborn Care, Oxfordshire, UK; ${ }^{33}$ College of Life Sciences, University of Leicester, Leicester, UK; ${ }^{34}$ Sickkids Research Institute, Toronto, ON, Canada; ${ }^{35}$ Indiana University School of
Medicine, Pediatrics, South Bend, IN, USA; ${ }^{36}$ Department of Pediatrics, University of Naples, Naples, Italy; ${ }^{37}$ Friedman Brain Institute, Pediatrics, Icahn School of Medicine at Mount Sinai, New York, NY, USA; ${ }^{38}$ Department of Medicine and Surgery, University of Salerno, Baronissi, Italy; ${ }^{39}$ Division of Pediatrics, UH Rainbow Babies and Children's Hospital, Cleveland, $\mathrm{OH}, \mathrm{USA} ;{ }^{40}$ University of Florida

College of Medicine, Gainesville, FL, USA and ${ }^{41}$ Paediatrics and Child Health, Trinity College, Trinity Academic Centre, Tallaght Hospital, The University of Dublin, Dublin, Ireland Correspondence: Cynthia Bearer (cbearer@som.umaryland.edu)

\section{ADDITIONAL INFORMATION}

Competing interests: The authors declare no competing interests.

Publisher's note Springer Nature remains neutral with regard to jurisdictional claims in published maps and institutional affiliations.

\section{REFERENCES}

1. Oberai, H. \& Anand, I. Unconscious bias: thinking without thinking. Human Resource Management International Digest 26, 14-17 (2018).

2. Lundine, J., Bourgeault, I. L., Clark, J., Heidari, S. \& Balabanova, D. The gendered system of academic publishing. Lancet 391, 1754-1756 (2018).

3. Bearer, C. F. \& Molloy, E. J. Gender bias at Pediatric Research? Pediatr. Res 86, 2 (2019).

4. Nielsen, M. W. et al. Opinion: gender diversity leads to better science. Proc. Natl. Acad. Sci. USA 114, 1740-1742 (2017).

5. Donald, A., Harvey, P. H. \& McLean, A. R. Athena SWAN awards: bridging the gender gap in UK science. Nature 478, 7367 (2011).

6. Jagsi, R., Rivkees, S. \& Opipari, V. P. An unbiased view about bias: not yet. Pediatr. Res. 86, 10-11 (2019).

7. Woolston, C. \& Osório, J. When English is not your mother tongue. Nature $\mathbf{5 7 0}$ 265-267 (2019). 